\title{
Comparison between Two Vertical Enclosures Filled With Porous Media under the Effect of Radiation and Magnetohydrodynamics
}

\author{
Hayder Ibrahim Mohammed ${ }^{1}$, Manal Hadi AL-Hafidh ${ }^{2}$ and Hussein Kalaf Jubair ${ }^{3}$ \\ 1. Department of Physics Education College, University of Garmian, Sulaymaniyah 964, Iraq \\ 2. Department of Mechanical Engineering, Faculty of Engineering, Baghdad University, Baghdad 964, Iraq \\ 3. Department of Energy Engineering, Faculty of Engineering, Baghdad University, Baghdad 964, Iraq
}

Received: May 16, 2013 / Accepted: July 02, 2013 / Published: January 31, 2014.

\begin{abstract}
A numerical study has been carried out to investigate the temperature distribution and the natural convection heat transfer in axisymmetric two-dimensional vertical saturated porous cylinder with steady state laminar flow. A comparison between two situations is done under the effect of MHD (magnetohydrodynamics) and radiation. In the two situations, the vertical walls of the cylinder are cooled with constant wall temperature and a constant heat generation subjected along the centerline of the cylinder. The first case for cylinder with insulated upper surface and cooled bottom surface while the second case for cylinder with cooled upper surface and insulated bottom surface. The governing equations used are continuity, momentum and energy equations which are transformed to dimensionless equations. The finite difference approach is used to obtain all the computational results using the MATLAB-7 programming. The parameters affected the system are Rayleigh number ranging within $\left(10^{2} \leq R a \leq 10^{4}\right)$, radiation parameter $(0 \leq R d \leq$ 2) and magnetohydrodynamics MHD $(M n)(0 \leq M n \leq 2)$. The results show that the temperature of Case 1 is more than that in Case 2 at constant $R a, M n$ and $R d$ while the value of the stream in Case 2 is greater than that in Case 1. $N u_{\text {mean }}$ increase with the increase of $R d$ and increasing $M n$ caused the temperature to increase and the streamline dropped while $N u_{\text {mean }}$ decreased. A correlation has been set up to give the average Nusselt number variation with $R a, R d$ and $M n$ for which the results are found to be in good agreement with previously published researches.
\end{abstract}

Key words: Radiation, magnetohydrodynamic, vertical enclosure, porous medium, laminar flow.

\section{Nomenclature}

Latin Symbols

Symbols Description

A Dimensionless aspect ratio $(A=H / R)$

$C_{p} \quad$ Specific heat at constant pressure

$g \quad$ Acceleration of gravity

$H \quad$ Axial length of cylinder

$h \quad$ Heat transfer coefficient

K Permeability

$k \quad$ Thermal conductivity

$\mathrm{Nu} \quad$ Nusselt number $(N u=h \cdot R / k)$
$N u_{\text {mean }} \quad$ Average Nusselt number

$N u_{\max }$

Nusselt number resulting from inverted -

Corresponding author: Manal Hadi AL-Hafidh, assistant professor, research fields: heat transfer, fluid mechanics and porous media. E-mail: manalhadi2005@yahoo.com. maximum temperature

Pressure N/m

Volumetric heat generation $\quad \mathrm{W} / \mathrm{m}^{3}$

Radiation flux $\quad \mathrm{W} / \mathrm{m}^{2}$

Temperature $\quad{ }^{\circ} \mathrm{C}$

Dimensionless temperature $\left(\bar{T}=\frac{2 k\left(T-T_{c}\right)}{q^{\circ} R^{2}}\right)$ -

Reference temperature $\quad{ }^{\circ} \mathrm{C}$

Cooled temperature for boundary $\quad{ }^{\circ} \mathrm{C}$

Dimensionless maximum temperature -

Dimensionless mean temperature

Radius of the cylinder

$\mathrm{m}$

Rayleigh number $\left(R a=\frac{K \rho g \beta q^{\circ} R^{3}}{2 \mu \alpha k}\right)$

Radiation parameter $\left(R d=\frac{4 \sigma_{i} T_{\infty}^{3}}{\beta k}\right)$

Radial coordinate

$\mathrm{m}$

Dimensionless radial coordinate $\left(\bar{r}=\frac{r}{R}\right) \quad$ - 


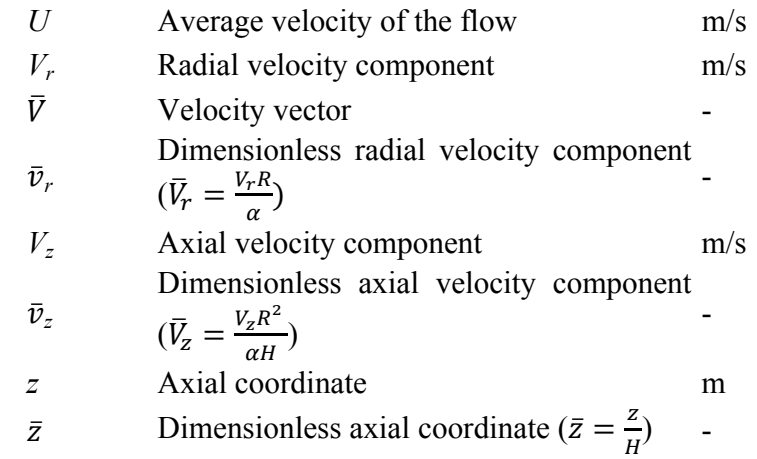

\section{Greek Symbols}

\begin{tabular}{|c|c|c|}
\hline Symbols & Description & Units \\
\hline$\alpha$ & Convective thermal diffusivity & $\mathrm{m}^{2} / \mathrm{s}$ \\
\hline$\beta$ & Volumetric thermal expansion coefficient & $1 / \mathrm{K}$ \\
\hline$\beta$ 。 & Strength of magnetic field & \\
\hline$\beta_{r}$ & Roseland extinction coefficient & $1 / \mathrm{m}$ \\
\hline$\varepsilon$ & Porosity & \\
\hline$\forall$ & Total volume of porous media & $\mathrm{m}^{3}$ \\
\hline$\forall_{p}$ & Solid matter volume & $\mathrm{m}^{3}$ \\
\hline$\forall$ 。 & Difference between $\forall \& \forall_{p}$ & $\mathrm{~m}^{3}$ \\
\hline$\sigma$ & Electrical conductivity of the fluid & - \\
\hline$\sigma_{i}$ & Stephan Boltzmann constant & $\mathrm{W} / \mathrm{m}^{2} \cdot \mathrm{K}^{4}$ \\
\hline$\Psi$ & Dimensionless Stream function & - \\
\hline$\gamma$ & Relaxation factor & - \\
\hline$\xi$ & Represents $\Psi$ and $\bar{T}$ & - \\
\hline$\psi$ & Stream function & $\mathrm{m}^{2} / \mathrm{s}$ \\
\hline$\Delta$ & Difference between two values & - \\
\hline$\mu$ & Dynamic viscosity & $\mathrm{kg} / \mathrm{m} \cdot \mathrm{s}$ \\
\hline$\nabla$ & $\begin{array}{l}\text { Laplacian in dimensionless cylindrical } \\
\text { coordinates }\end{array}$ & \\
\hline$\eta$ & $\begin{array}{l}\text { Actual value of the quantity of a point } \\
\text { inside the sample volume }\end{array}$ & - \\
\hline$\rho_{\circ}$ & Reference density at $T$ 。 & $\mathrm{kg} / \mathrm{m}^{3}$ \\
\hline$\phi_{p}$ & Value of the state variable at node & - \\
\hline
\end{tabular}

\section{Introduction}

Various heat transfer mechanisms and geometries have been studied by some engineers and scientists purposely to augment heat transfer in heat exchangers and some other heat transfer equipments. A numerical investigation of natural convection was studied for vertical annulus. Prasad and Kulacki [1] studied numerically free convection in a vertical annulus filled with saturated porous media whose vertical walls at constant temperatures and insulated horizontal wall. Prasad and Kulacki [2] studied numerically and experimentally the free convection heat transfer in short cylindrical annuli filled with saturated porous media. For an annulus whose inner wall heated with constant temperature and outer wall isothermally cooled, the top and the bottom being insulated. Prasad and Chui [3] investigated the natural convection inside a cylindrical enclosure filled with a volumetrically heated, saturated porous medium. Three cases were studied (a) both top and bottom adiabatic; (b) insulated bottom and cooled top; and (c) both top and bottom were cooled, and the vertical wall was cooled for all cases. Shivakumara and Prasanna [4] studied numerically the free convection in vertical cylinder annulus filled with fluid saturated porous medium with the inner wall heated to a uniform temperature, the outer wall cooled to a uniform temperature and maintaining top and bottom boundaries at adiabatic conditions. Rashad [5] studied the magnetohydrodynamics and radiation effect in laminar boundary layer flow of Newtonian viscous fluid over a vertical flat plate embedded in a fluid saturated porous media in the presence of thermophoresis particle deposition effect. Saxena and Dubey [6] studied the effects of MHD free convection heat and mass transfer flow of viscoelastic fluid embedded in a porous medium of variable permeability with radiation effect and heat source in slip flow regime. Unsteady MHD heat and mass transfer free convection flow of polar fluids past a vertical moving porous plate in a porous medium with heat generation and thermal diffusion was analyzed by Ref. [7]. Reddy et al. [8] considered the effect of radiation and chemical reaction on free convection MHD flow through a porous medium bounded by vertical surface.

The present study considers the investigation of the temperature distribution and the natural convection heat transfer in axisymmetric two-dimensional vertical saturated porous cylinder with steady state laminar flow. A comparison between two situations is done under the effect of MHD and radiation. In the two situations the vertical walls of the cylinder are cooled with constant wall temperature and a constant heat generation subjected along the centerline of the 
cylinder. The first case for cylinder with insulated upper surface and cooled bottom surface while the second case for cylinder with cooled upper surface and insulated bottom surface.

\section{Mathematical Model}

The schematic drawing of the geometry and the Cartesian coordinate system employed in solving the problem is shown in Fig. 1. The fluid flows naturally in these cylinders. These cylinders is symmetrical about z-axis $(\partial / \partial r=0)$. Convection heat transfer through a saturated porous media with a fluid (liquid or gas) is based on a series of concepts; such concepts include porosity and permeability of the porous media, and the volume average properties of the fluid flowing through the medium.

The governing equations for axisymmetric, steady flow through Darcy porous medium including the radiation and magnetohydrodynamic effect are:

\subsection{Continuity Equation}

$$
\frac{1}{r} \frac{\partial}{\partial r}\left(r V_{r}\right)+\frac{\partial V_{z}}{\partial z}=0
$$

\subsection{Momentum Equations}

$$
\begin{gathered}
V_{z}=-\frac{K}{\mu}\left[\frac{\partial p}{\partial z}+\rho g+\sigma \beta_{\circ}^{2} V_{z}\right] \text { in } \mathrm{Z} \text { direction (2) } \\
V_{r}=-\frac{K}{\mu}\left[\frac{\partial p}{\partial r}+\sigma \beta_{\circ}^{2} V_{r}\right] \text { in R direction }
\end{gathered}
$$

The density of the fluid assumed constant except when it is happened directly from flotation force as in Ref. [9].

$$
\left.\begin{array}{c}
\rho=\rho_{\circ}\left[1-\beta\left(T-T_{\circ}\right)\right] \\
\beta=\frac{1}{\rho}\left(\frac{\partial \rho}{\partial T}\right)
\end{array}\right\}
$$

From Eqs. (2) and (4):

In $\mathrm{Z}$ direction:

$$
V_{z}=-\frac{K}{\mu}\left[\frac{\partial p}{\partial z}+\rho_{\circ} g\left[1-\beta\left(T-T_{\circ}\right)\right]+\sigma \beta_{\circ}^{2} V_{z}\right]
$$

\subsection{Energy Equation}

$$
\begin{gathered}
V_{r} \frac{\partial T}{\partial r}+V_{z} \frac{\partial T}{\partial z}=\frac{k}{\rho c_{p}}\left[\frac{1}{r} \frac{\partial}{\partial r}\left(r \frac{\partial T}{\partial r}\right)+\frac{\partial}{\partial z}\left(\frac{\partial T}{\partial z}\right)-\frac{1}{r} \frac{\partial}{\partial r}\left(\frac{r q_{r}}{k}\right)\right]+\frac{q^{\circ}}{\rho c_{p}}(6) \\
q_{r}=-\frac{4 \sigma_{\mathrm{i}} \partial T^{4}}{3 \beta_{\mathrm{r}} \partial r}
\end{gathered}
$$

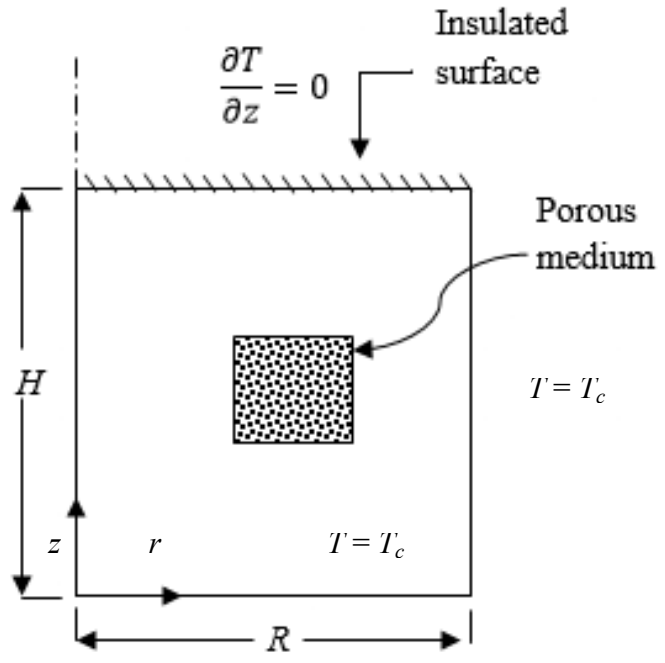

(a) Case 1

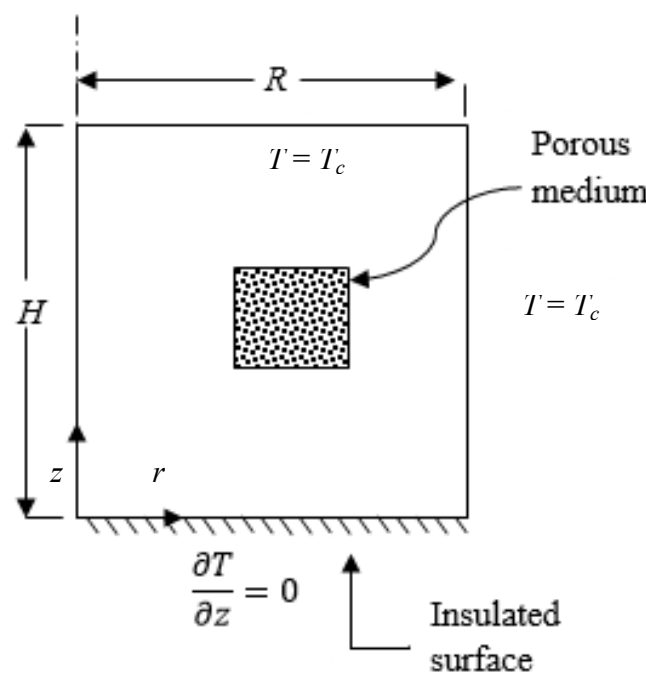

(b) Case 2

Fig. 1 Geometric configuration, coordinate system and the boundary conditions.

\section{Normalization Parameters}

The variables in the governing equations and boundary conditions are transformed to dimensionless formula by employing the following transformation parameters:

$$
\begin{gathered}
\bar{r}=\frac{r}{R}, \bar{z}=\frac{z}{H}, \bar{T}=\frac{T-T_{c}}{\frac{q^{2} R^{2}}{2 k}}, \bar{V}_{z}=\frac{V_{z} R^{2}}{H \alpha} \\
\bar{V}_{r}=\frac{V_{r} R}{\alpha}, \bar{V}_{z}=\frac{1}{\bar{r}} \frac{\partial \psi}{\partial \bar{r}}, \bar{V}_{r}=-\frac{1}{\bar{r}} \frac{\partial \psi}{\partial \bar{z}}
\end{gathered}
$$

By using the relation above Eqs. (3), (5) and (6) become:

$$
\left[\frac{\partial}{\partial \bar{r}}\left(\frac{1}{\bar{r}} \frac{\partial \Psi}{\partial \bar{r}}\right)+\frac{\partial}{\partial \bar{z}}\left(\frac{1}{\bar{r}} \frac{\partial \Psi}{\partial \bar{z}}\right)\right]=\frac{R a}{1+M n}\left[\frac{\partial \bar{T}}{\partial \bar{r}}\right]
$$




$$
\begin{array}{r}
{\left[-\left(\frac{1}{\overline{\mathrm{r}}} \frac{\partial \Psi}{\partial \bar{z}} \frac{\partial \bar{T}}{\partial \bar{r}}\right)+\left(\frac{1}{\overline{\mathrm{r}}} \frac{\partial \Psi}{\partial \bar{r}} \frac{\partial \bar{T}}{\partial \bar{z}}\right)\right]=} \\
{\left[\left(1+\frac{4}{3} R d\right) \frac{1}{\overline{\mathrm{r}}} \frac{\partial}{\partial \bar{r}}\left(\bar{r} \frac{\partial \bar{T}}{\partial \bar{r}}\right)+\left(\frac{\partial^{2} \bar{T}}{\partial \overline{\mathrm{z}}^{2}}\right)+2\right]}
\end{array}
$$

The boundary conditions of Case 1 are:

$$
\left.\begin{array}{l}
\bar{r}=0, \Psi=0, \frac{\partial \bar{T}}{\partial \bar{r}}=0 \\
\bar{r}=1, \Psi=0, \bar{T}=0 \\
\bar{z}=0, \Psi=0, \frac{\partial \bar{T}}{\partial \bar{z}}=0 \\
\bar{z}=1, \Psi=0, \bar{T}=0
\end{array}\right\}
$$

The boundary conditions of Case 2 are:

$$
\left.\begin{array}{c}
\bar{r}=0, \Psi=0, \frac{\partial \bar{T}}{\partial \bar{r}}=0 \\
\bar{r}=1, \Psi=0, \bar{T}=0 \\
\bar{z}=0, \Psi=0, \bar{T}=0 \\
\bar{z}=1, \Psi=0, \frac{\partial \bar{T}}{\partial \bar{z}}=0
\end{array}\right\}
$$

\section{Overall Heat Transfer}

For the problem considered here, there is no well-defined characteristic temperature difference to express the heat transfer coefficient. However, one can define an overall Nusselt number based on either the maximum temperature in the cylinder or the mean temperature in the centerline. When the heat transfer coefficient is defined in terms of $\left(\bar{T}_{\max }-\bar{T}_{c}\right)$, the overall Nusselt number is defined as in Ref. [3].

$$
N u_{\max }=\frac{h R}{k}=\frac{1}{\bar{T}_{\max }}
$$

$N u_{\max }$ is Nusselt number which means inverted maximum temperature, and not mean maximum Nusselt number. In the similar way, the average Nusselt number based on the mean temperature difference $\left(\bar{T}_{\text {mean }}-\bar{T}_{c}\right)$ can be expressed as:

$$
N u_{\text {mean }}=\frac{1}{\bar{T}_{\text {mean }}}
$$

\section{Numerical Solution}

The new algebra equations system can be solved using some of known techniques, like relaxation method, to give approximate values of the dependent variables at a number of discrete points called (grid points or nodes) in the computational domain. A grid was established by subdividing the computational domain in the $\mathrm{R}$ and $\mathrm{Z}$ directions with indexes $i$ and $j$ that are integers describing the number of radial grid lines from the center of the cylinder and the number of axial grid lines from the bottom to upper surface respectively. The spacing of the grid lines in the R-direction is uniform and given by $(\Delta r)$ and that of the grid lines in the $\mathrm{Z}$-direction is also uniform and given by $(\Delta z)$. The number of the grid points will be $[m \times n]$ where $(m)$ represents the number of gridlines in the R-direction and equals $[(1 / \Delta \bar{r})+1]$ while $(n)$ represents the number of gridlines in the Z-direction and equals $[(1 / \Delta \bar{z})+1]$.

The partial differential Eqs. (9) and (10) were finite-differenced using central difference schemes for all of the derivatives. In particular, let $\xi$ represents $\Psi$ and $\bar{T}$ then:

$$
\left.\begin{array}{c}
\Delta \bar{r}=\Delta \bar{z}, \Delta \overline{\mathrm{r}}_{\mathrm{i}=} \Delta \overline{\mathrm{r}}_{\mathrm{i}+1}, \Delta \overline{\mathrm{z}}_{\mathrm{j}=} \Delta \overline{\mathrm{z}}_{\mathrm{j}+1} \\
\frac{\partial \xi}{\partial \overline{\mathrm{r}}}=\frac{\xi_{(\mathrm{i}+1, \mathrm{j})}-\xi_{(\mathrm{i}-1, \mathrm{j})}}{2 \Delta \overline{\mathrm{r}}}, \frac{\partial \xi}{\partial \mathrm{z}}=\frac{\xi_{(\mathrm{i}, \mathrm{j}+1)}-\xi_{(\mathrm{i}, \mathrm{j}-1)}}{2 \Delta \overline{\mathrm{z}}} \\
\frac{\partial^{2} \xi}{\partial \overline{\mathrm{r}}^{2}}=\frac{\xi_{(\mathrm{i}+1, \mathrm{j})}-2 \xi_{(\mathrm{i}, \mathrm{j})}+\xi_{(\mathrm{i}-1, \mathrm{j})}}{\Delta \overline{\mathrm{r}}^{2}} \\
\frac{\partial^{2} \xi}{\partial z^{2}}=\frac{\xi_{(i, j+1)}-2 \xi_{(i, j)}+\xi_{(i, j-1)}}{\Delta \bar{z}^{2}}
\end{array}\right\}
$$

In terms of the above expressions momentum and energy equations become:

\subsection{Momentum Equation}

$$
\begin{gathered}
\frac{1}{\bar{r}_{(i)}} \frac{\Psi_{(\mathrm{i}+1, \mathrm{j})}-2 \Psi_{(\mathrm{i}, \mathrm{j})}+\Psi_{(\mathrm{i}-1, \mathrm{j})}}{\Delta \bar{r}^{2}}-\frac{1}{\bar{r}_{(i)}^{2}} \frac{\Psi_{(\mathrm{i}+1, \mathrm{j})}-\Psi_{(\mathrm{i}-1, \mathrm{j})}}{2 \Delta \overline{\mathrm{r}}}+ \\
\frac{\Psi_{(\mathrm{i}, \mathrm{j}+1)}-2 \Psi_{(\mathrm{i}, \mathrm{j})}+\Psi_{(\mathrm{i}, \mathrm{j}-1)}}{\bar{r}_{(i)} \Delta \overline{\mathrm{z}}^{2}}=\frac{R a}{1+M n} \frac{\overline{\mathrm{T}}_{(\mathrm{i}+1, \mathrm{j})}-\overline{\mathrm{T}}_{(\mathrm{i}-1, \mathrm{j})}}{2 \Delta \overline{\mathrm{r}}}
\end{gathered}
$$

\subsection{Energy Equation}

$$
\begin{array}{r}
\frac{1}{\bar{r}_{(i)}} \cdot \frac{\Psi_{(\mathrm{i}+1, \mathrm{j})}-\Psi_{(\mathrm{i}-1, \mathrm{j})}}{2 \Delta \overline{\mathrm{r}}} \cdot \frac{\overline{\mathrm{T}}_{(\mathrm{i}, \mathrm{j}+1)}-\overline{\mathrm{T}}_{(\mathrm{i}, \mathrm{j}-1)}}{2 \Delta \overline{\mathrm{z}}}- \\
\frac{1}{\overline{\mathrm{r}}_{(i)}} \cdot \frac{\overline{\mathrm{T}}_{(\mathrm{i}+1, \mathrm{j})}-\overline{\mathrm{T}}_{(\mathrm{i}-1, \mathrm{j})}}{2 \Delta \overline{\mathrm{r}}} \cdot \frac{\Psi_{(\mathrm{i}, \mathrm{j}+1)}-\Psi_{(\mathrm{i}, \mathrm{j}-1)}}{2 \Delta \overline{\mathrm{z}}}= \\
\left(1+\frac{4}{3} R d\right)\left[\frac{\overline{\mathrm{T}}_{(\mathrm{i}+1, \mathrm{j})}-2 \overline{\mathrm{T}}_{(\mathrm{i}, \mathrm{j})}+\overline{\mathrm{T}}_{(\mathrm{i}-1, \mathrm{j})}}{\Delta \overline{\mathrm{r}}^{2}}+\frac{1}{\overline{\mathrm{r}}_{(i)}} \frac{\overline{\mathrm{T}}_{(\mathrm{i}+1, \mathrm{j})}-\overline{\mathrm{T}}_{(\mathrm{i}-1, \mathrm{j})}}{2 \Delta \overline{\mathrm{r}}}\right]+ \\
\frac{\overline{\mathrm{T}}_{(\mathrm{i}, \mathrm{j}+1)}-2 \overline{\mathrm{T}}_{(\mathrm{i}, \mathrm{j})}+\overline{\mathrm{T}}_{(\mathrm{i}, \mathrm{j}-1)}}{\Delta \overline{\mathrm{z}}^{2}}+2
\end{array}
$$




\section{Relaxation Method}

As a solution method for the system of algebraic (8) and (9) obtained from the discretization of governing differential equations, iterative relaxation method was used. In relaxation methods, the value of the variable to be used for obtaining the solution in the next iteration is the value in the current iteration plus a fraction of the difference between the current value and the predicted value as follows:

$$
\phi_{p}^{\zeta+1}=\phi_{p}^{\zeta}+\gamma\left(\frac{\sum\left(a_{\zeta \mathrm{b}} * \phi_{\zeta b}\right)+b}{a_{p}}-\phi_{p}^{\zeta}\right)
$$

where:

$\gamma>0$ is the relaxation factor;

$\phi_{p}^{\zeta+1}$ is the value of the state variable at node $P$ to be used for the next iteration;

$\phi_{p}^{\zeta}$ is the value of the state variable at node $P$ form current iteration;

$\phi_{\zeta b}$ are the values of the variable at the surrounding nodes and $a, p$ and $b$ are the constants from the discretized equation.

The grid that used in both cases was $(41 \times 61)$ for $R a$ $=10^{2}$ and $10^{3}$, and $(81 \times 81)$ for $R a=10^{4}$. Generally, the temperature is over relaxed and/or the stream function is under relaxed.

\section{Results and Discussion}

The results of numerical modeling have been compared with the notable previously published studies. The results obtained from the present study give a good agreement when compared with Ref. [2] where maximum deviation was found to be $2.85 \%$ and with Ref. [3] where the maximum deviation was found to be $2.25 \%$. Tables 1 and 2 show the value of the mean $N u$ of the present work compared with previous work, with clarification of percent deviation. Where $\vartheta$ is the value of $\left(r_{o}-r_{i}\right)$ in Ref. [2].

\subsection{Centerline Temperature Distribution}

Figs. 2-4 show the effect of $R d, M n$ and $R a$ on the centerline temperature distribution for the both cases, Fig. 2 shows the temperature variation along the
Table 1 Comparison of the present results with that of Ref. [2].

\begin{tabular}{lllll}
\hline \multirow{v}{*}{} & $R a$ & $N u$. Ref. [2] & $\begin{array}{l}\text { Nu. present } \\
\text { study }\end{array}$ & \%Dev. \\
\hline \multirow{2}{*}{2.5} & 90 & 4.271 & 4.30 & -0.679 \\
& 30 & 2.275 & 2.21 & 2.857143 \\
3.333 & 105 & 5.071 & 5.01 & 1.202919 \\
& 35 & 2.691 & 2.62 & 2.638424 \\
5 & 120 & 6.242 & 6.3 & -0.92919 \\
& 40 & 3.384 & 3.36 & 0.70922 \\
\hline
\end{tabular}

Table 2 Comparison of the present results with that of Ref. [2].

\begin{tabular}{|c|c|c|c|}
\hline$A=1$ & $\begin{array}{l}\boldsymbol{N u} \boldsymbol{u}_{\text {Mean }} \\
\text { Ref. [2] }\end{array}$ & $\begin{array}{l}\mathbf{N} \boldsymbol{u}_{\text {Mean }} \\
\text { present study }\end{array}$ & $\%$ Dev. \\
\hline$R a=100$ & 3.155 & 3.226 & -2.2504 \\
\hline$R a=1,000$ & 7.639 & 7.61 & 0.379631 \\
\hline$R a=10,000$ & 19.841 & 19.433 & 2.056348 \\
\hline
\end{tabular}

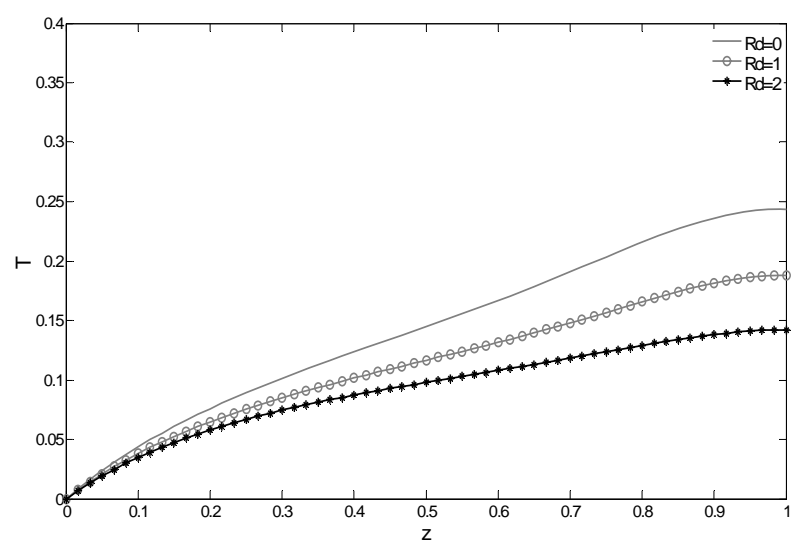

(a) Case 1

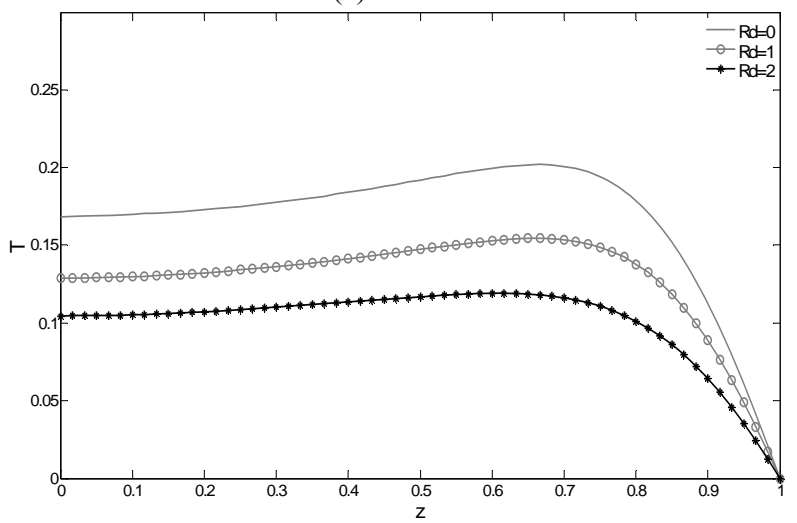

(b) Case 2

Fig. 2 Effect of $R d$ on the temperature distribution at the centerline of the cylinders for $(R a=1,000$ and $M n=2)$.

cylinder centerline with increasing of the radiation parameter. Because heat diffusion in the system increase with increasing of $R d$, the results for Case 1 show that the centerline temperature is decrease with increasing of radiation and the maximum dimensionless 


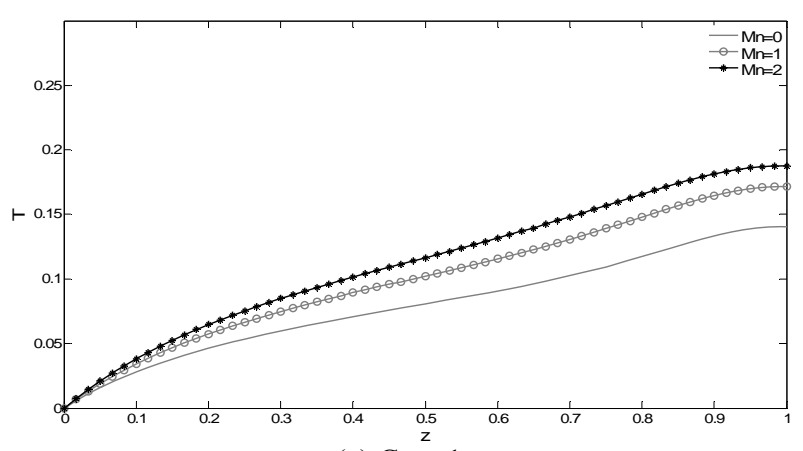

(a) Case 1

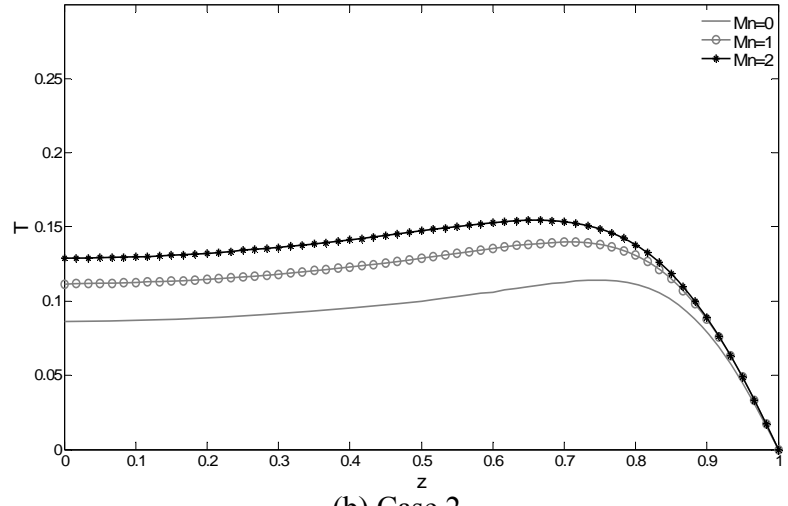

(b) Case 2

Fig. 3 Effect of $M n$ on the temperature distribution at the centerline of the cylinders for $(R a=1,000$ and $R d=2)$.

temperature is 0.243 at $R d=0$ adjacent to the upper surface, because the hot fluid gathered there, and the maximum centerline temperature in Case 1 is greater than that of Case 2. In Case 2, the upper surface is cold therefore the hot fluid gathered in the upper half side relatively far from the upper surface and the maximum temperature is 0.2 at $R d=0$.

The temperature in the centerline increase with increasing of the MHD parameter as it is clear in Fig. 3 this is because the force which created from MHD acting in the opposite direction of the force that caused the motion of the fluid and this behavior caused a low diffusion of the heat and so cause to increase the temperature, and again the maximum temperature for Case 1 is greater than that in Case 2.

Fig. 4 illustrates that the dimensionless temperature in the centerline dropped with the increasing of $R a$, because of the circulation occurred in the flow which cause more heat diffusion, and also like other two previous parameters the maximum centerline temperature in Case 1 is greater than that in Case 2.

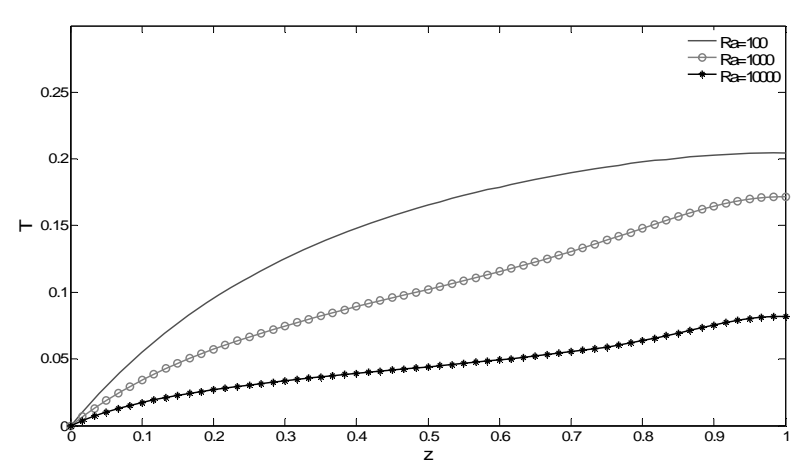

(a) Case 1

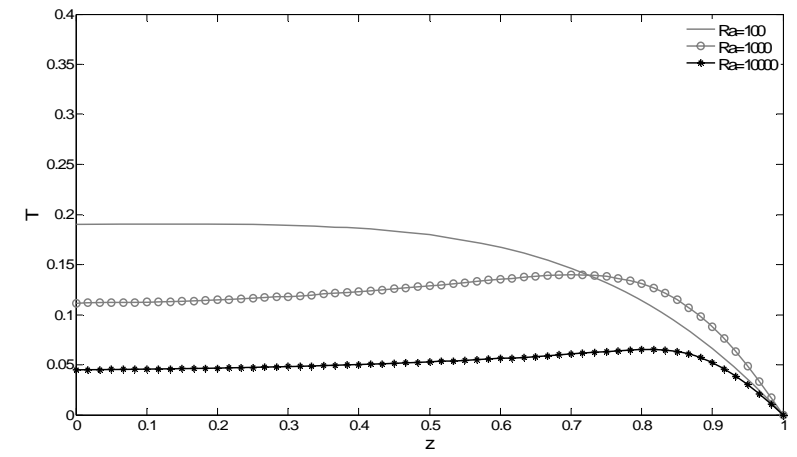

(b) Case 2

Fig. 4 Effect of $R a$ on the temperature distribution at the centerline of the cylinders for $(R d=1$ and $M n=1)$.

\subsection{Streamlines and Temperature Fields}

\subsubsection{The Effect of Radiation Parameter $R d$}

The $R d$ effect on the temperature distribution for Case 1 and Case 2 at $(R a=1,000$ and $M n=1)$ is presented in Fig. 5. Case 1 is illustrated in Figs. 5a, 5c and $5 \mathrm{e}$ where the warm region area restricted between the centerline and the upper wall of the cylinder and the cold region was adjacent to the cold vertical and bottom walls. The maximum value for isothermal contours is (0.1963) at $R d=0$ and when $R d$ increase the warm region is contracted from the upper wall and more expand in the centerline observed. For Case 2, Figs. 5b, $5 \mathrm{~d}$ and $5 \mathrm{f}$ the upper surface is cold so the hot region of the fluid can not gather adjacent to the upper surface therefore it is gather in the upper half side of the cylinder relatively far from the upper wall, so the average temperatures in Case 1 is more than that in Case 2.

Fig. 6 shows the effect of $R d$ on streamlines. It is clear from Fig. 6 that the change in $R d$ do not affect on the behavior of the flow too much. When Rd increases the system loss more heat so the stream value decrease. 


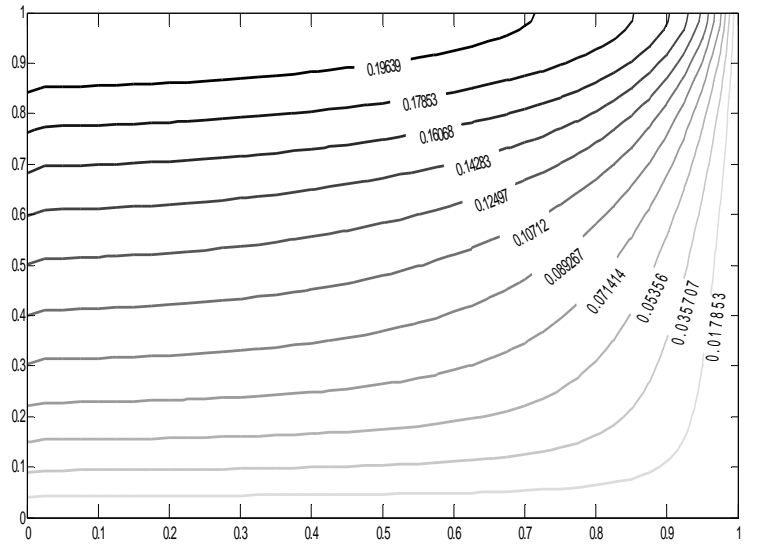

(a) Case $1, R d=0$

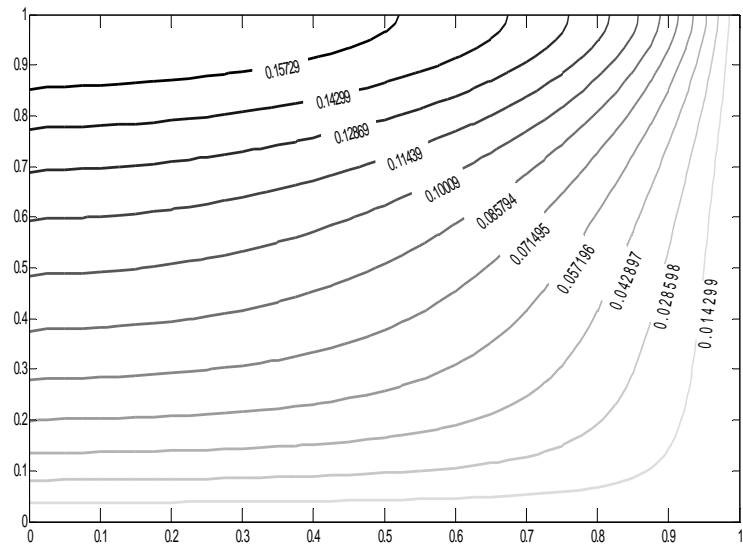

(c) Case $1, R d=1$

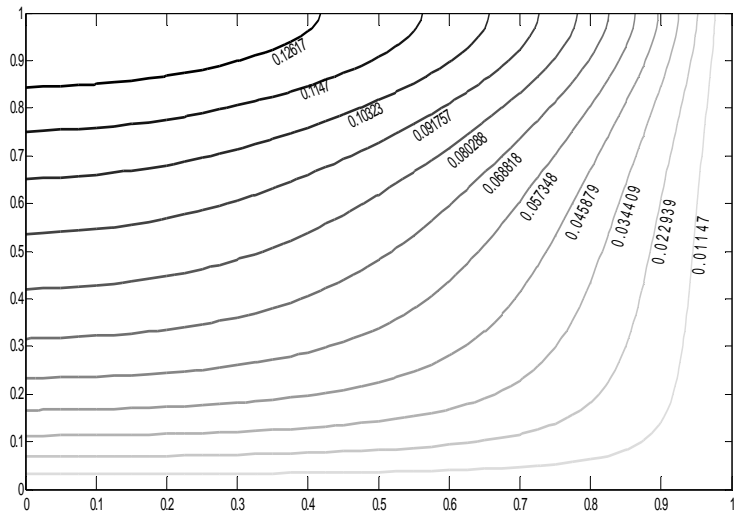

(e) Case $1, R d=2$

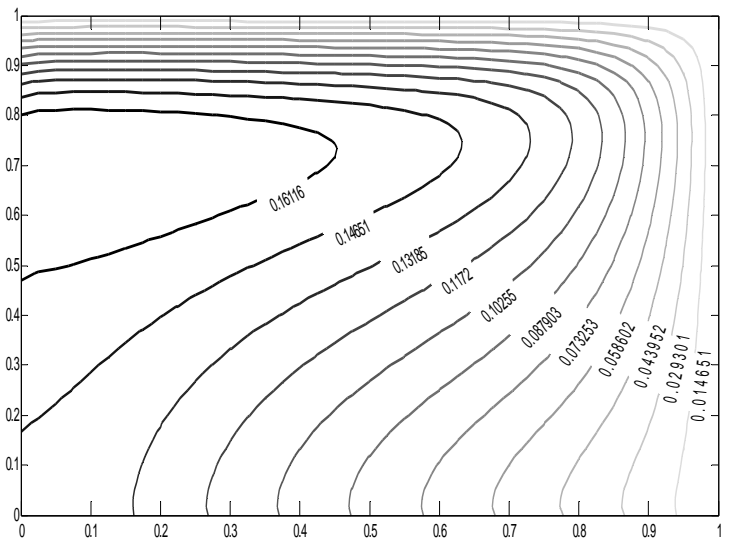

(b) Case 2, $R d=0$

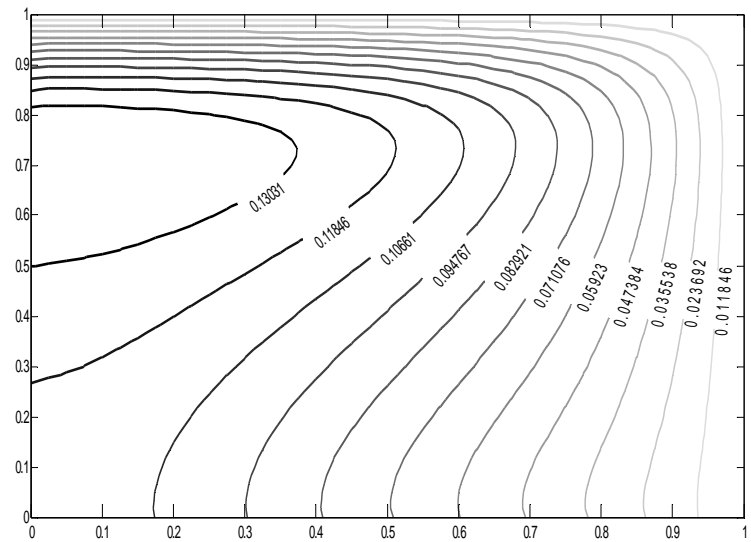

(d) Case 2, $R d=1$

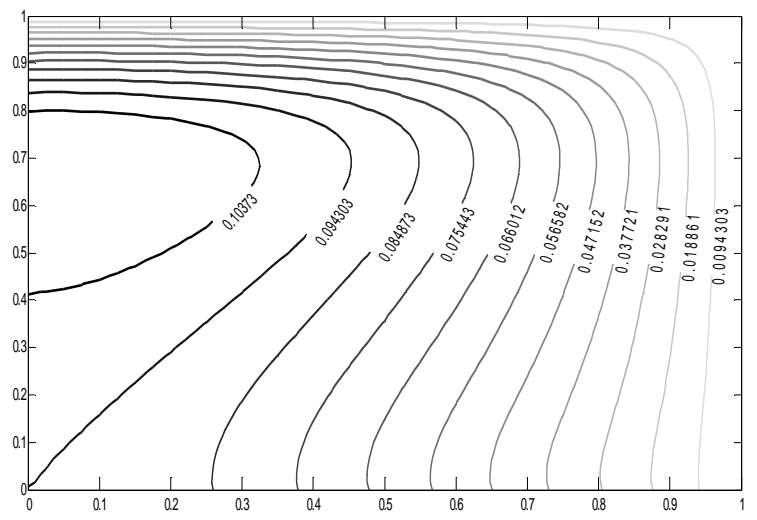

(f) Case 2, $R d=2$

Fig. 5 Effect of $R d$ on the temperature distribution for $R a=1,000$ and $M n=1$.

The streamline value in Case 2 is more than that in Case 1 for any value of $R d$, for example, when $R d=0$ the stream value is 2.768 in Case 1 as shown in Fig. 6a, and 3.247 in Case 2 as it is clear in Fig. $6 \mathrm{~b}$, when $R d=1$ the stream value is 2.36 in Case 1, Fig. 6c and 2.7216 in Case 2, Fig. 6d.

7.2.2 The Effect of Magnetohydrodynamic $M n$

To study the effect of MHD the parameters taken in Fig. 7 are $R a=10,000, R d=1$. This figure illustrates the temperature distribution, for Case 1 in Figs. 7a, 7c and $7 \mathrm{e}$ with the upper surface insulated, the warm region is adjacent to the upper surface and when $\mathrm{Mn}$ increases the average temperature of the system increase. For Case 2, when $M n=0$ the warm region concentrate far away from the upper cold wall with $T_{\max }=0.045$. In Fig. $7 \mathrm{~b}$ when the value of $M n$ increases the temperature of the hotter region increase too. 


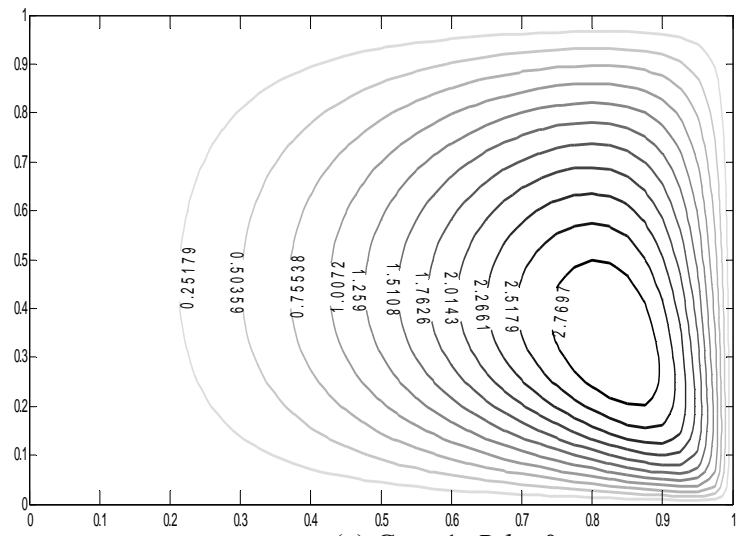

(a) Case $1, R d=0$

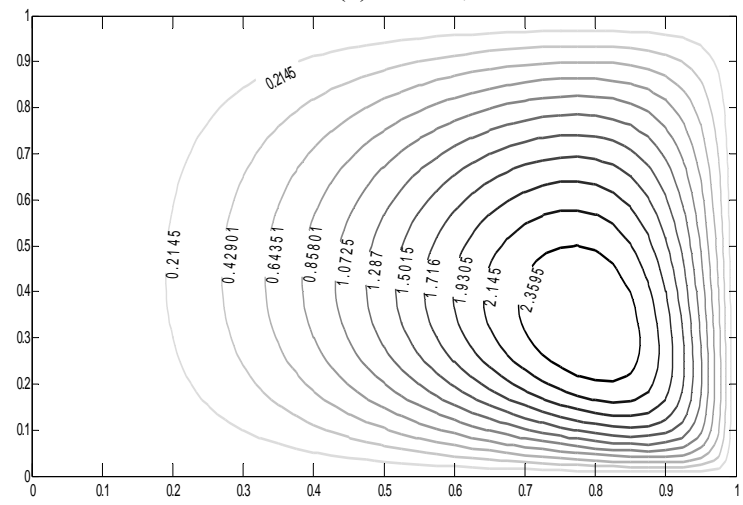

(c) Case $1, R d=1$

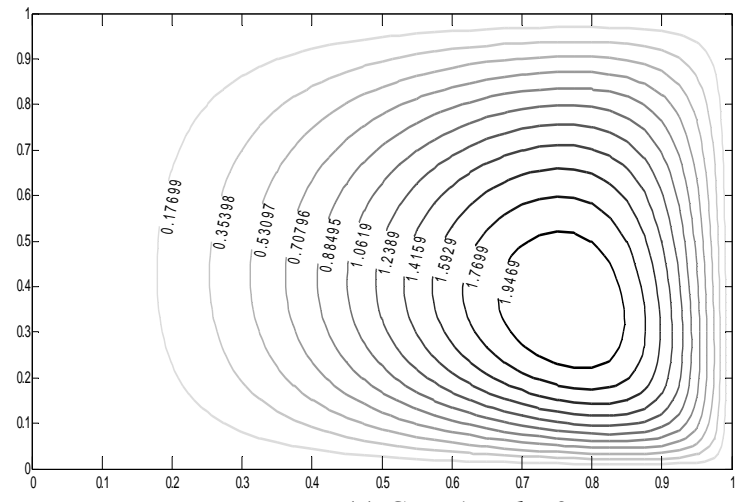

(e) Case $1, R d=2$

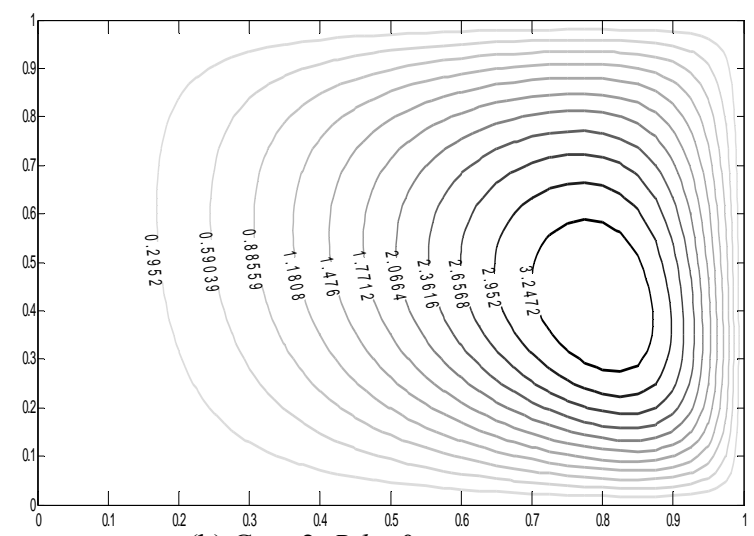

(b) Case 2, $R d=0$

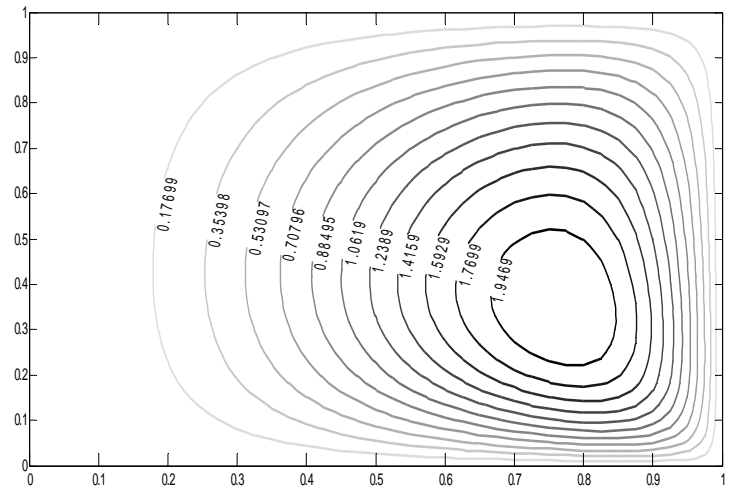

(d) Case2, $R d=1$

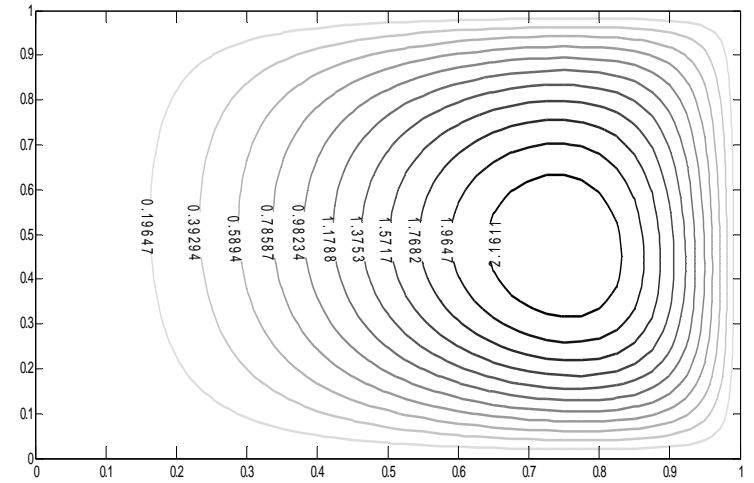

(f) Case 2, Rd $=2$

Fig. 6 Effect of $R d$ on the fluid flow contours for $R a=1,000$ and $M n=1$.

\subsubsection{The Effect of Rayleigh Number $R a$}

The effect of Rayleigh number $R a$ on the temperature distribution and streamlines for both cases at $(R d=2, M n=2)$ is presented in Figs. 8 and 9 respectively. From Fig. 8a, it is clear for Case 1 that when $R a=100$, the hotter region concentrate adjacent to the upper wall and center of cylinder with a value of 0.132 , by increasing $R a$ to 1,000 as in Fig. $8 \mathrm{c}$ the hotter region will be 0.13 and come close to the upper wall and for $R a$ equal 10,000 as in Fig. 8e the hotter region will decrease to 0.078 , for Case 2 the average temperature also decrease with the increase of $R a$, so when $R a=100$ in Fig. 8b, the warmer region value is 0.113 and adjacent to bottom wall and the center of the cylinder, and increasing $R a$ cause the warmer region to rise up and move away from the insulated bottom wall Figs. 8d and $8 f$. 


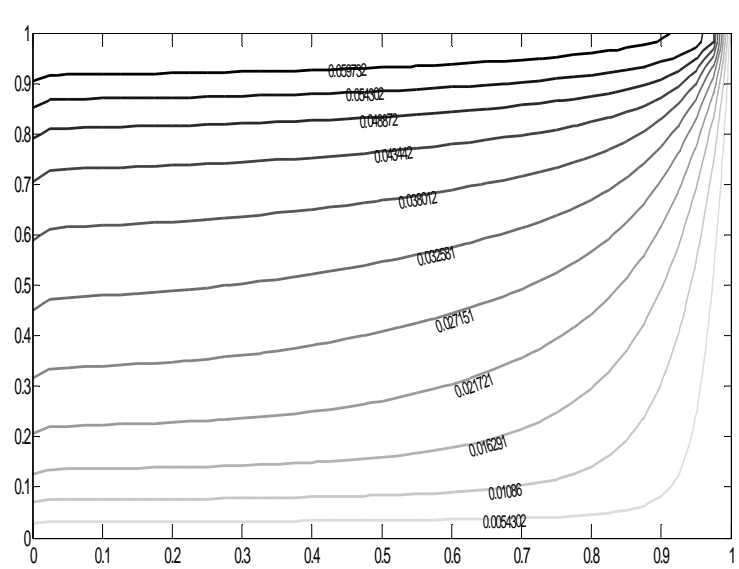

(a) Case $1, M n=0$

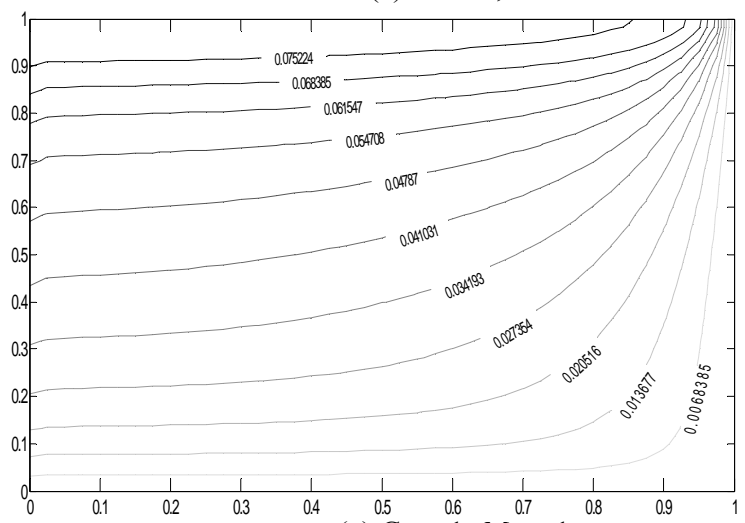

(c) Case $1, M n=1$

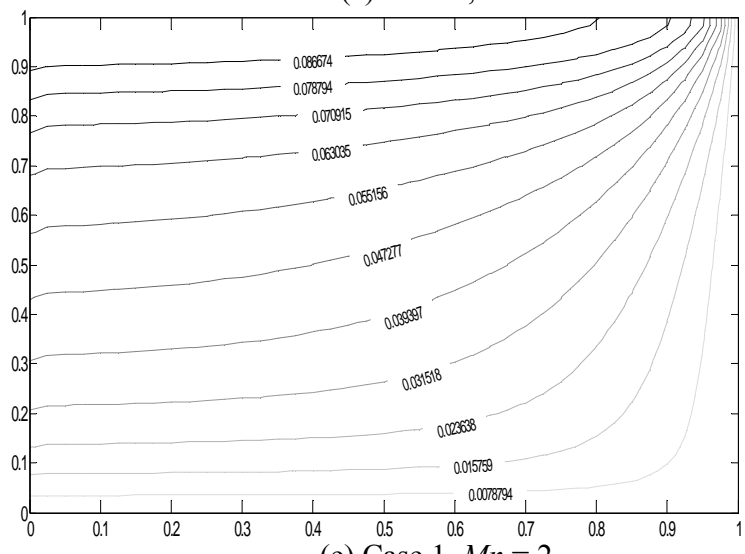

(e) Case $1, M n=2$

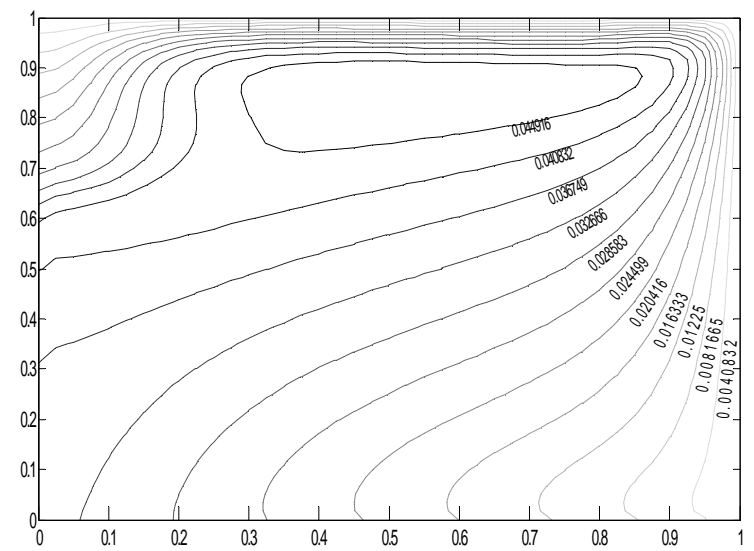

(b) Case 2, $M n=0$

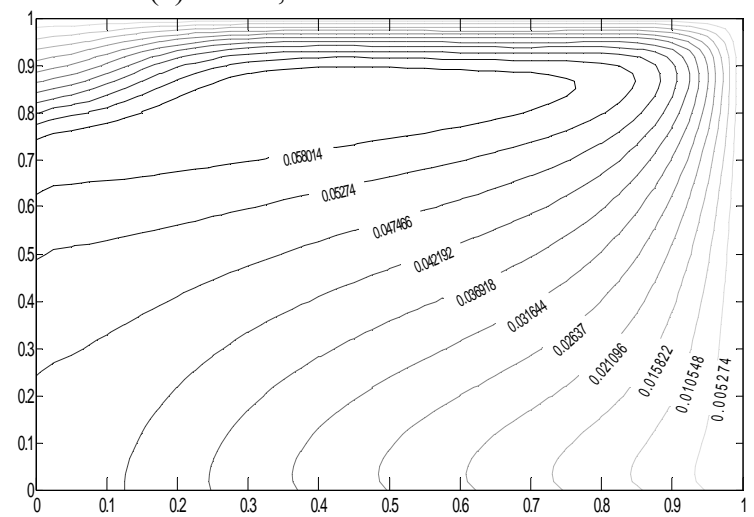

(d) Case 2, Mn = 1

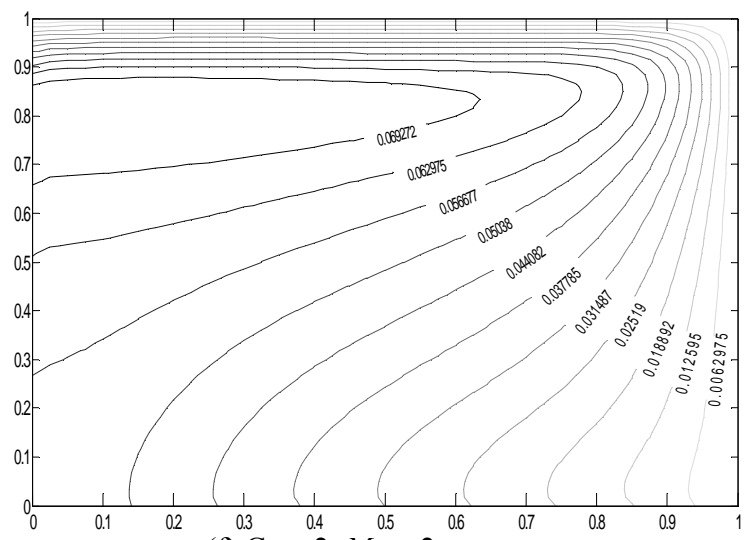

(f) Case 2, $M n=2$

Fig. 7 Effect of $M n$ on the temperature contours for $R a=10,000$ and $R d=1$.

In Fig. 9, it is clear that the streamline value in the internal cell increase with the increase of $R a$ for both cases. The figure illustrates that the value of the stream in Case 2 is larger than that for Case 1, this behavior was happened because the increase of $R a$ cause to increase the velocity and more circulation between the layers of the fluid will be occurred that caused the temperature to be dropped and the stream value increase.

\subsubsection{Mean Nusselt Number $N u_{\text {mean }}$}

Fig. 10 illustrates the variation of $N u_{\text {mean }}$ with $M n$ for $R d=1$ and for different values of $R a$. It is shown that for both cases, for $R a$ equal 10 , the value of $N u_{\text {mean }}$ almost is constant and for $R a \geq 100, N u_{\text {mean }}$ decreases with the increase of $M n$. Comparing both cases in Fig. 10 illustrate that $N u_{\text {mean }}$ in Case 1 is greater than that in Case 2. 


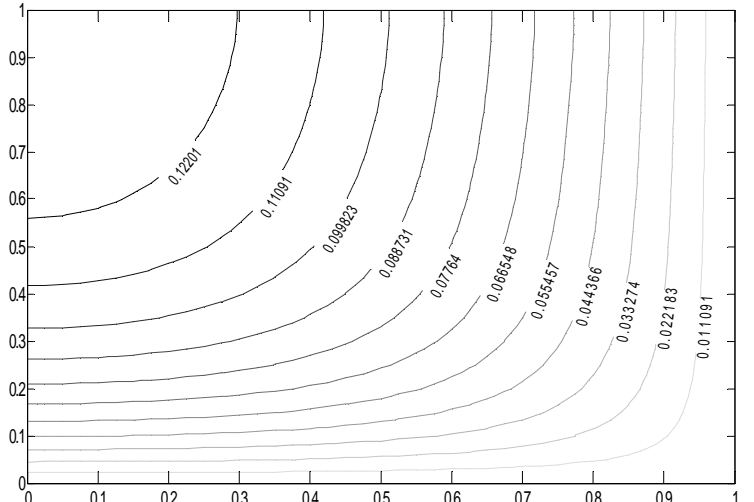

(a) Case $1, R a=100$

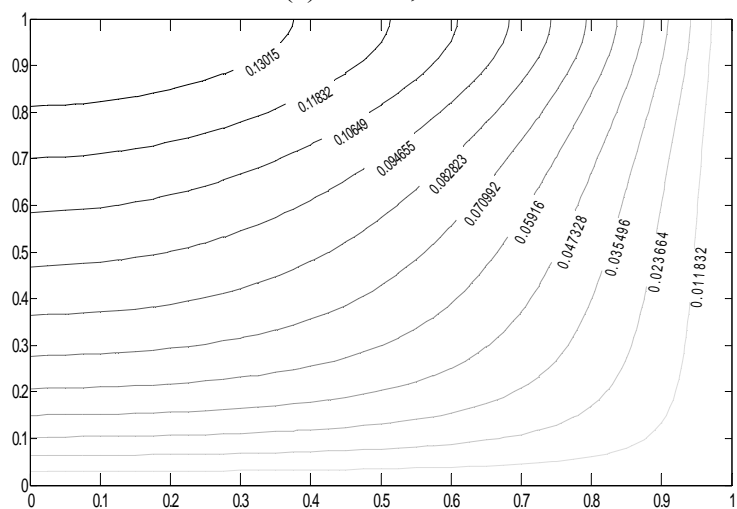

(c) Case $1, R a=1,000$

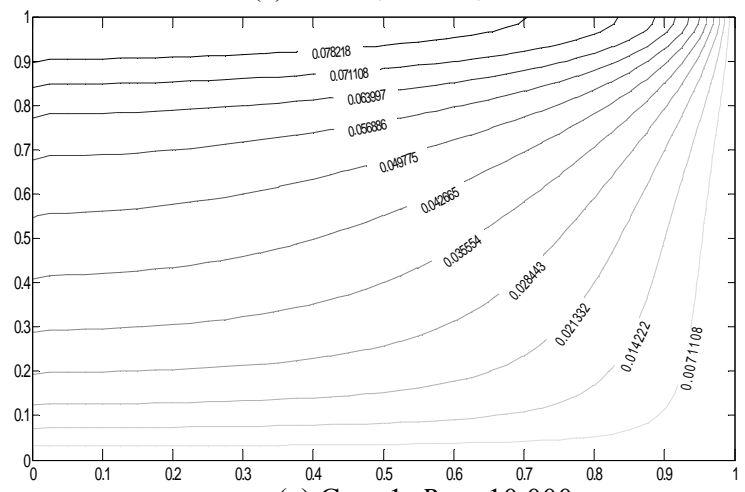

(e) Case $1, R a=10,000$

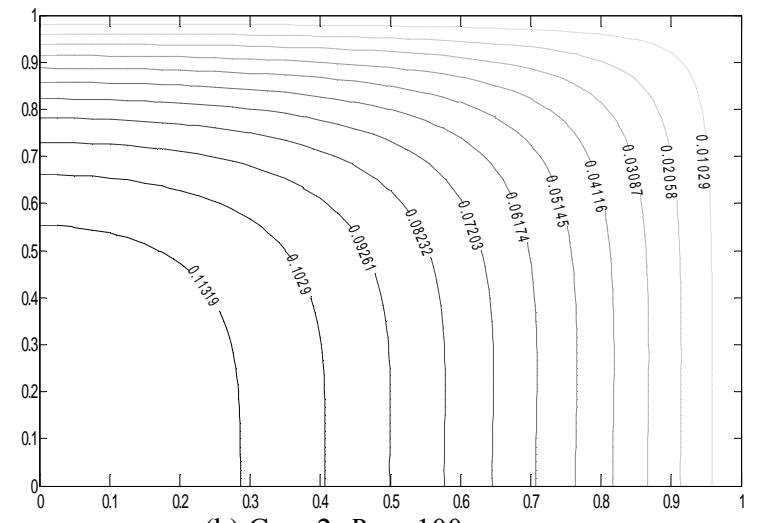

(b) Case 2, $R a=100$

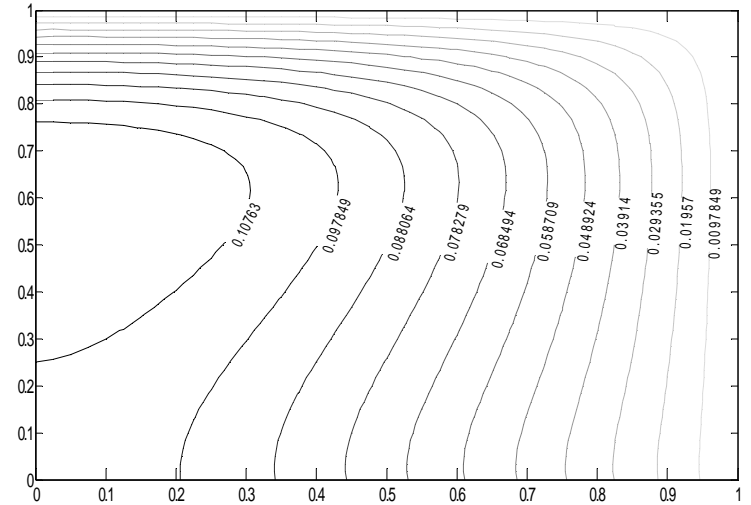

(d) Case 2, $\mathrm{Ra}=1,000$

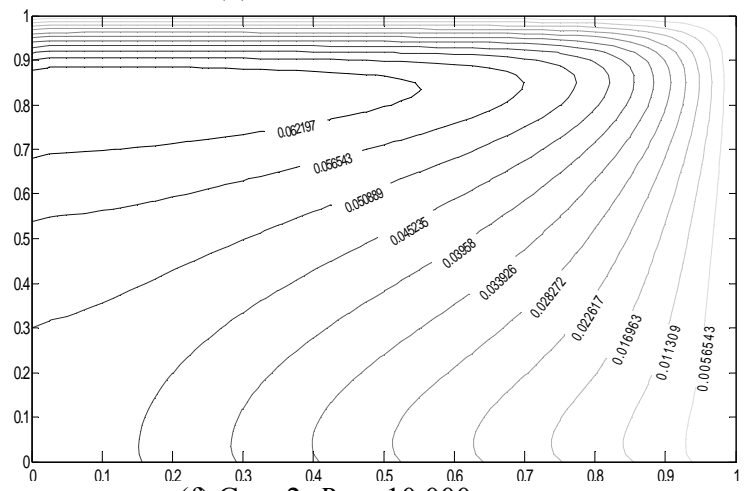

(f) Case 2, $R a=10,000$

Fig. 8 Effect of $R a$ on the temperature contours $R d=2$ and $M n=2$.

Fig. 11 shows the variation of $N u_{\text {mean }}$ with $M n$ at $R a$ $=1,000$ for different values of $R d$. For both cases, the value of $N u_{\text {mean }}$ decrease with the increase of $M n$ and increase with the increase of $R d$ and comparing the values of $N u_{\text {mean }}$ for both cases show that it is greater than that for Case 2 at any value of $M n$.

Fig. 12 illustrates the variation of $N u_{\text {mean }}$ with $R d$ at $R a=100$ for different values of $M n$. From part (a) (Case 1) of the last figure we see that the value of the mean Nusselt number $N u_{\text {mean }}$ decrease with the increase of $M n$ at a certain value of $R d$, and for the certain value of $\mathrm{Mn}, \mathrm{Nu} u_{\text {mean }}$ increase with increasing $R d$. Compared with the part (b) of the last figure that illustrate Case 2, it is seen that the $N u_{\text {mean }}$ in the Case 1 is more than Case 2 at any value of $R d$ under the same conditions.

\section{Conclusions}

From the present work results the following conclusions can be obtained: 


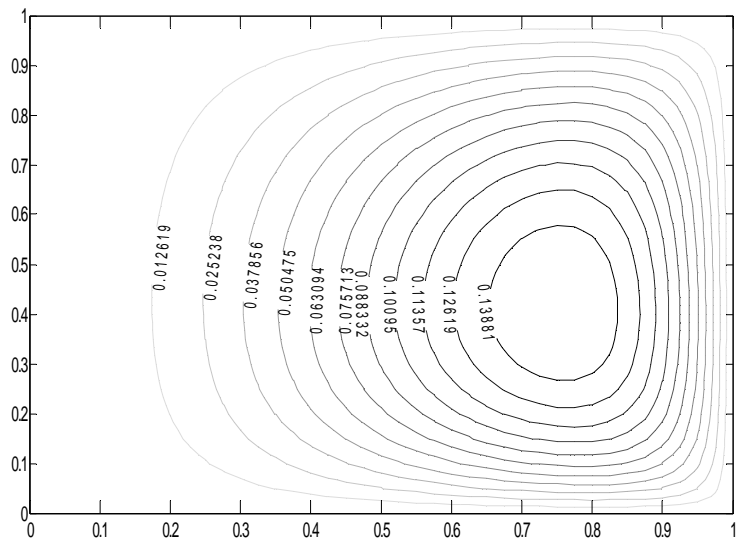

(a) Case $1, R a=100$

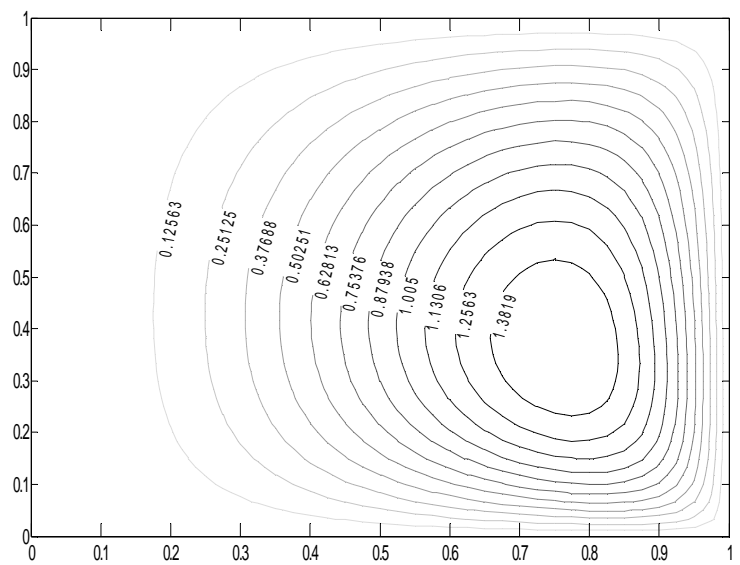

(c) Case $1, R a=1,000$

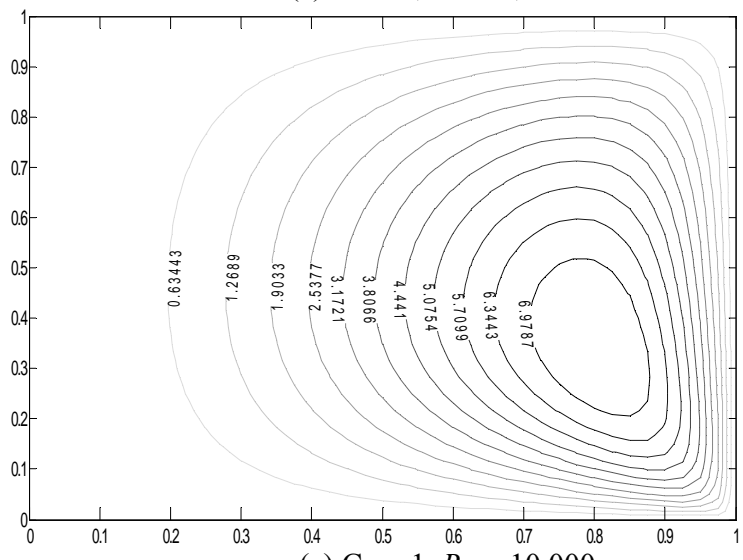

(e) Case $1, R a=10,000$

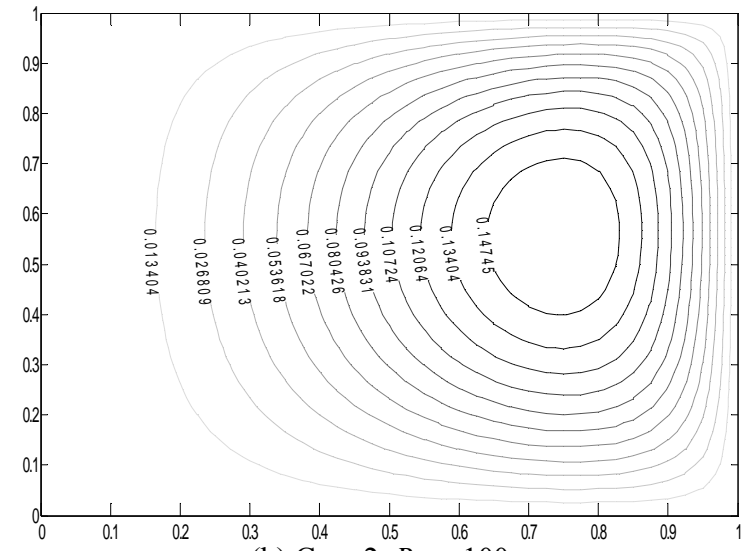

(b) Case 2, $R a=100$

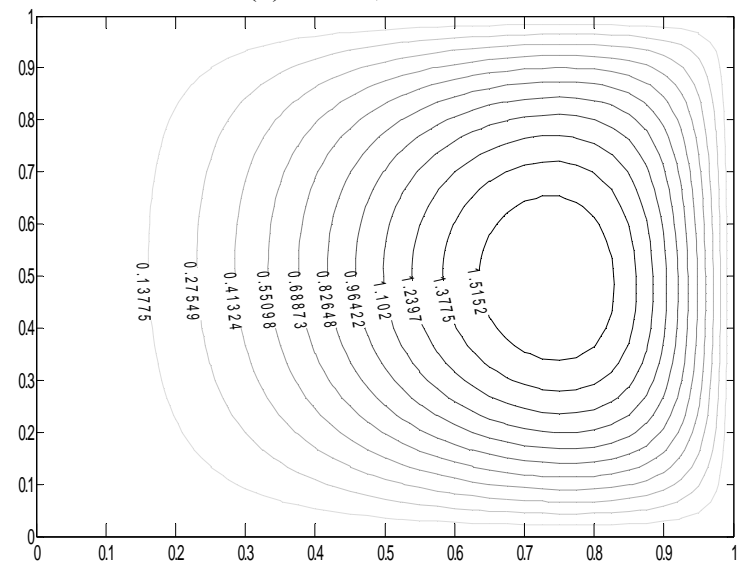

(d) Case 2, $R a=1,000$

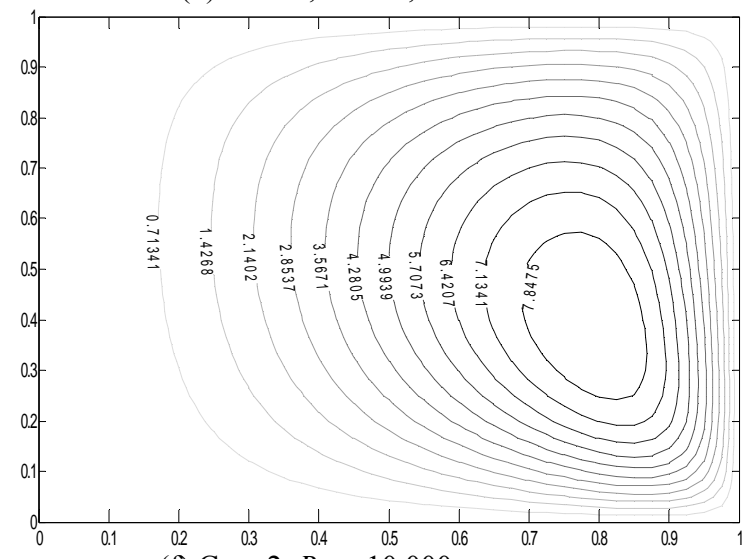

(f) Case 2, $R a=10,000$

Fig. 9 Effect of $R a$ on the streamlines contours for $R d=2$ and $M n=2$.

For both cases, for $R a$ equal 10 , the value of $N u_{\text {mean }}$ almost is constant and for $R a \geq 100, N u_{\text {mean }}$ decreases with the increase of $M n$.

The centerline temperature decreases with increasing of radiation and the maximum dimensionless temperature is 0.243 at $R d=0$ adjacent to the upper surface, because the hot fluid gathered there, and the maximum centerline temperature in Case 1 is greater than that of Case 2 .

The dimensionless temperature in the centerline dropped with the increasing of $R a$, because of the circulation occurred in the flow which cause more heat diffusion, and also like other two previous parameters the maximum centerline temperature in Case 1 is 

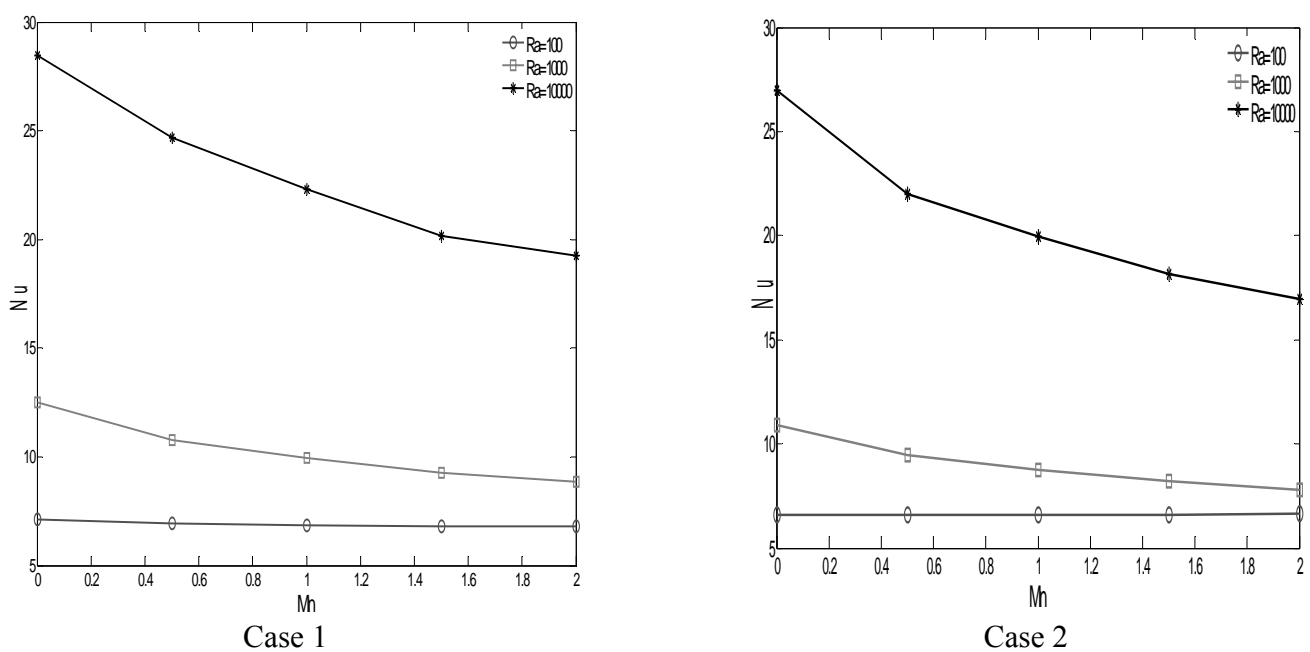

Case 2

Fig. 10 The variation of $N u_{\text {mean }}$ with $M n$ at $R d=1$ for different values of $R a$.
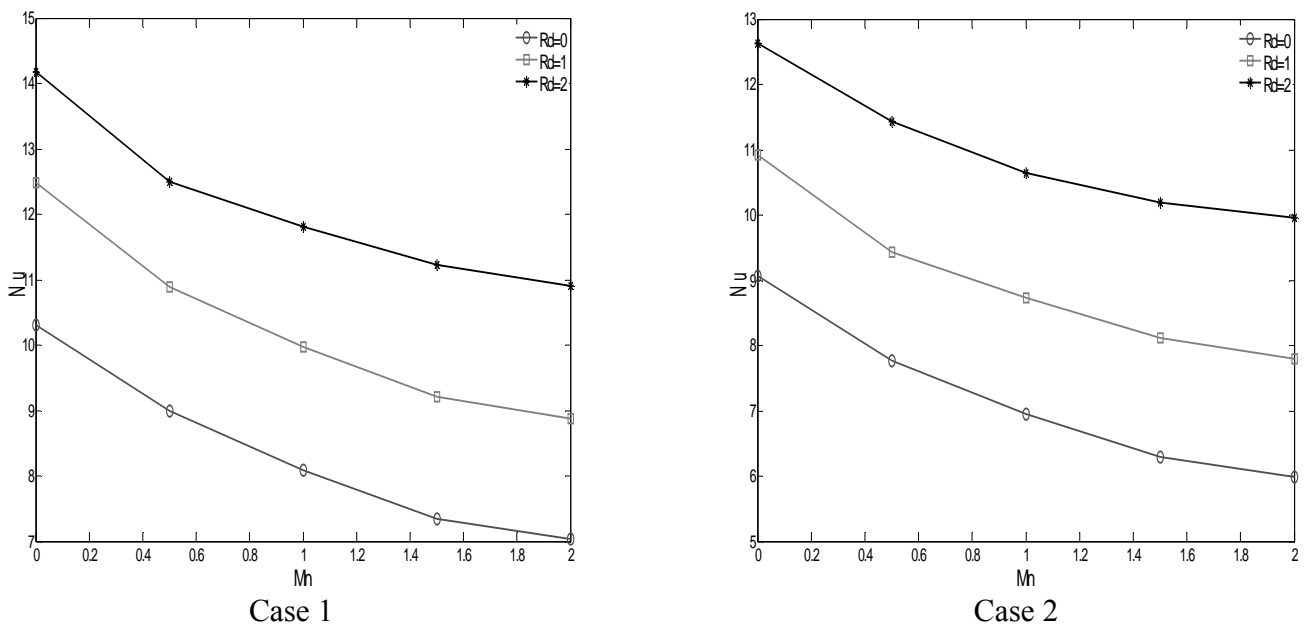

Fig. 11 The variation of $N u_{\text {mean }}$ with $M n$ at $R a=1,000$ for different values of $R d$.

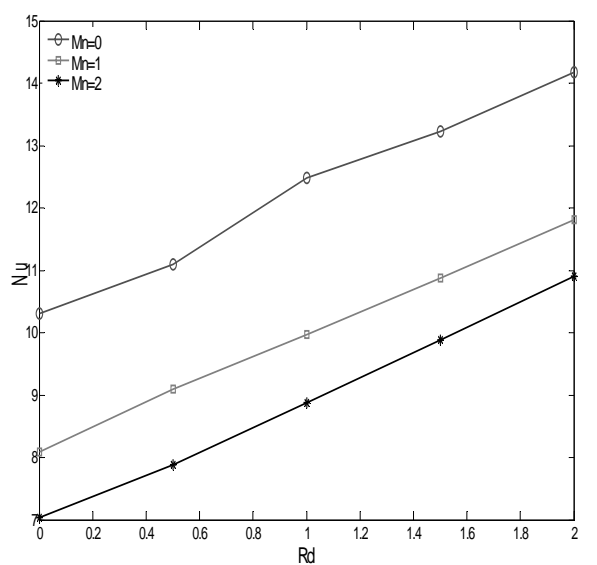

Case 1

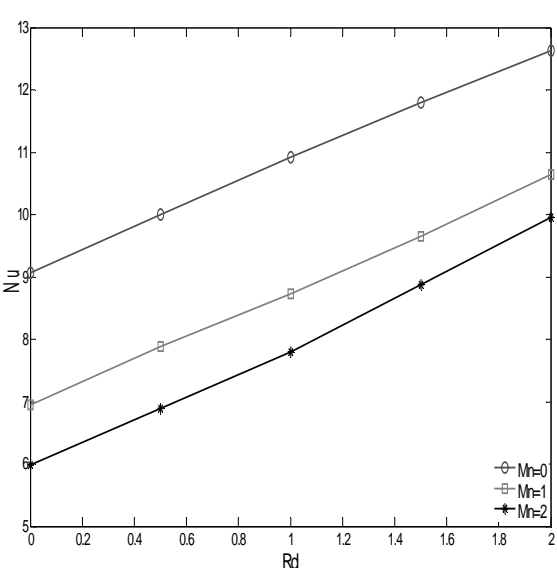

Case 2

Fig. 12 The variation of $N u_{\text {mean }}$ with $R d$ at $R a=100$ for different values of $M n$.

greater than that in Case 2.

The results obtained from the present study give a good agreement when compared with Ref. [2] where maximum deviation was found to be $2.85 \%$ and with Ref. [3] where the maximum deviation was found to be $2.25 \%$. 


\section{Effect of Radiation and Magnetohydrodynamics}

\section{References}

[1] V. Prasad, F.A. Kulacki, Natural convection in a vertical porous annulus, Int. J. Heat Mass Transfer 27 (2) (1984) 207-219.

[2] V. Prasad, F.A. Kulacki, Natural convection in porous media bounded by short concentric vertical cylinders, Transaction of ASME, J. of Heat Transfer 107 (1985) 147-154.

[3] V. Prasad, A. Chui, Natural convection in cylindrical porous enclosure with internal heat generation, Transaction of ASME, J. of Heat Transfer 111 (1989) 916-925.

[4] I.S. Shivakumara, B.M.R. Prasanna, Numerical study of natural convection in a vertical cylindrical annulus using non-Darcy equation, J. of Porous Media 5 (2) (2002) 87-102.

[5] A.M. Rashad, Influence of radiation on MHD free convection from a vertical flat plate embedded in porous media with thermophoretic deposition of particles, Communication in Nonlinear Science and Numerical Simulation 13 (2007) 2213-2222.

[6] S.S. Saxena, G.K. Dubey, MHD free convection heat and mass transfer flow of viscoelastic fluid embedded in a porous medium of variable permeability with radiation effect and heat source in slip flow regime, Advances in Applied Science Research 2 (5) (2011) 115-129.

[7] S.S. Saxena, G.K. Dubey, Unsteady MHD heat and mass transfer free convection flow of polar fluids past a vertical moving porous plate in a porous medium with heat generation and thermal diffusion, Advances in Applied Science Research 2 (4) (2011) 259-278.

[8] S. Reddy, G.V.R. Reddy, Radiation and chemical reaction effects on free convection MHD flow through a porous medium bounded by vertical surface, Advances in Applied Science Research 3 (3) (2012) 1603-1610.

[9] A. Bejan, D. Nield, Convection in Porous Media, Springer, 1999. 